\title{
Chromosomal microarray analysis, including constitutional and neoplastic disease applications, 2021 revision: a technical standard of the American College of Medical Genetics and Genomics (ACMG)
}

Lina Shao ${ }^{1}$, Yassmine Akkari ${ }^{2}$, Linda D. Cooley ${ }^{3,4}$, David T. Miller ${ }^{5}$, Bryce A. Seifert ${ }^{6}$, Daynna J. Wolff ${ }^{7}$, Fady M. Mikhail ${ }^{8}$ and ACMG Laboratory Quality Assurance Committee ${ }^{9 *}$

Disclaimer: This technical standard is designed primarily as an educational resource for clinical laboratory geneticists to help them provide quality clinical laboratory genetic services. Adherence to this technical standard is voluntary and does not necessarily assure a successful medical outcome. This technical standard should not be considered inclusive of all proper procedures and tests or exclusive of other procedures and tests that are reasonably directed to obtaining the same results. In determining the propriety of any specific procedure or test, the clinical laboratory geneticist should apply his or her own professional judgment to the specific circumstances presented by the individual patient or specimen.

Clinical laboratory geneticists are encouraged to document in the patient's record the rationale for the use of a particular procedure or test, whether or not it is in conformance with this technical standard. They also are advised to take notice of the date any particular technical standard was adopted, and to consider other relevant medical and scientific information that becomes available after that date. It would also be prudent to consider whether intellectual property interests may restrict the performance of certain tests and other procedures.

Chromosomal microarray technologies, including array comparative genomic hybridization and single-nucleotide polymorphism array, are widely applied in the diagnostic evaluation for both constitutional and neoplastic disorders. In a constitutional setting, this technology is accepted as the first-tier test for the evaluation of chromosomal imbalances associated with intellectual disability, autism, and/or multiple congenital anomalies. Furthermore, chromosomal microarray analysis is recommended for patients undergoing invasive prenatal diagnosis with one or more major fetal structural abnormalities identified by ultrasonographic examination, and in the evaluation of intrauterine fetal demise or stillbirth when further cytogenetic analysis is desired. This technology also provides important genomic data in the diagnosis, prognosis, and therapy of neoplastic disorders, including both hematologic malignancies and solid tumors. To assist clinical laboratories in the validation of chromosomal microarray methodologies for constitutional and neoplastic applications, the American College of Medical Genetics and Genomics (ACMG) Laboratory Quality Assurance Committee has developed these updated technical laboratory standards, which replace the ACMG technical standards and guidelines for microarray analysis in constitutional and neoplastic disorders previously published in 2013.

Genetics in Medicine (2021) 23:1818-1829; https://doi.org/10.1038/s41436-021-01214-w

\section{GENERAL CONSIDERATIONS}

Purpose of chromosomal microarray testing

Cytogenetic abnormalities include numerical abnormalities (aneuploidy, hypodiploidy, hyperdiploidy, and polyploidy) and structural abnormalities (deletion, duplication, triplication, amplification, translocation, inversion, insertion, marker chromosome, etc.). The chromosomal microarray (CMA) platforms discussed in these technical standards are those designed for the detection of DNA copy-number gains and losses associated with unbalanced chromosomal aberrations. In addition, regions of homozygosity $(\mathrm{ROH})$, also referred to as copy-neutral loss of heterozygosity $(\mathrm{CN}-\mathrm{LOH})$, regions with absence of heterozygosity $(\mathrm{AOH})$, or long continuous stretches of homozygosity (LCSH), may also be detected by platforms that include single-nucleotide polymorphism (SNP)-detecting probes.

The CMA diagnostic yield for detection of germline copynumber changes in patients with developmental delay, intellectual disability, autism, and/or multiple congenital anomalies has

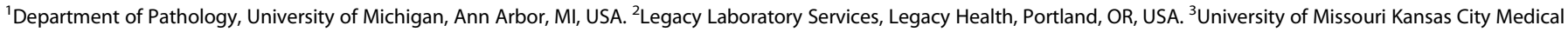

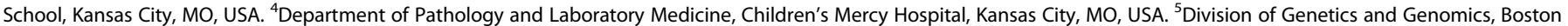

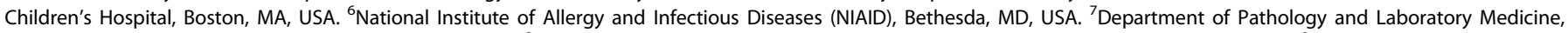

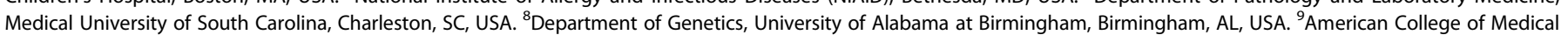

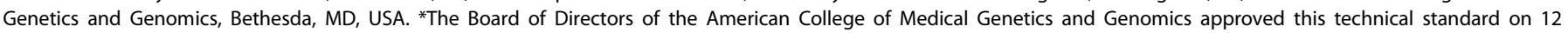
April 2021. ${ }^{\circledR}$ email: documents@acmg.net
} 
been well documented, and CMA is recommended by the American College of Medical Genetics and Genomics (ACMG) as the first-tier test for these indications. ${ }^{1,2}$ Similarly, CMA is recommended by the American College of Obstetricians and Gynecologists (ACOG) and the Society for Maternal-Fetal Medicine (SMFM) as the first-tier test in a prenatal setting in patients undergoing invasive prenatal diagnosis with one or more major fetal structural abnormalities identified by ultrasonographic examination, and for the evaluation of intrauterine fetal demise or stillbirth when further cytogenetic analysis is desired. ${ }^{3}$ In addition, CMA is recommended as a follow-up test for small copynumber changes that are reported by noninvasive prenatal screening (NIPS). ${ }^{4}$

In a neoplastic setting, cytogenetic analysis plays important roles in the diagnosis, prognosis, and therapy of many neoplastic disorders. G-banded chromosome and/or fluorescence in situ hybridization (FISH) analyses are the gold standard for detection of clinically significant chromosomal abnormalities in many neoplasms. ${ }^{5}$ Published clinically applicable data now show the clinical utility of CMA in the assessment of multiple neoplastic disorders, both hematologic malignancies and solid tumors. ${ }^{6-11}$ Examples of the clinical utility of CMA in both hematologic malignancies and solid tumors can be found in the ACMG/Cancer Genomics Consortium (CGC) technical laboratory standards for interpretation and reporting of acquired copy-number abnormalities and CN-LOH in neoplastic disorders. ${ }^{12}$

The current technical standards serve as an update of the ACMG technical standards and guidelines for CMA analysis in constitutional disorders, including postnatal and prenatal applications and neoplastic disorders, that were published in $2013 .^{13,14}$ In an attempt to standardize the terminology used to communicate clinical CMA results, the workgroup reached consensus on the definitions of specific terms shown below:

- Copy-number variant (CNV): This term is used to describe germline copy-number gain and/or loss of chromosomal material.

- Copy-number abnormality (CNA): This term is used to describe acquired copy-number gain and/or loss of chromosomal material in neoplastic disorders.

- Copy-number change: This term is used to describe germline and acquired copy-number gain and/or loss of chromosomal material (both CNV and CNA).

- Region of homozygosity (ROH): This term is used to describe a region with germline allelic imbalance (i.e., homozygosity) without an associated copy-number change.

- Copy-neutral loss of heterozygosity (CN-LOH): This term is used to describe a region with acquired allelic imbalance (i.e., homozygosity) without an associated copy-number change in neoplastic disorders.

\section{Advantages of CMA}

The advantages of the use of CMA include the ability to:

- Use any sample that yields DNA of sufficient quality and quantity.

- Detect abnormalities that are cytogenetically cryptic by standard G-banded chromosome analysis.

- Better define and characterize abnormalities detected by standard G-banded chromosome analysis.

- Customize the CMA platform to concentrate probes in areas of interest.

- Interpret objective data, rather than a subjective visual assessment of band intensities.

- Detect ROH and CN-LOH using CMA platforms incorporating SNP probes.

- Interface the data with genome browsers and databases.
Limitations of CMA

The limitations of the use of CMA include the inability to detect:

- Genetic events that do not affect the relative copy-number of DNA sequences (e.g., molecularly balanced chromosomal rearrangements); however, CMA may reveal copy-number changes in apparently "balanced" chromosomal rearrangements (i.e., gains or losses at or near the chromosomal breakpoint sites).

- Low-level mosaicism for unbalanced rearrangements and aneuploidy in a constitutional setting, and inability to detect tumor-specific changes (acquired clonality) in a small percentage of cells. CMA analysis is neither established nor recommended as a method for post-therapy follow-up or for minimal residual disease detection in a neoplastic setting, unless an aberration is only detected by CMA (e.g., CN-LOH). The sensitivity of CMA for detection of mosaicism and acquired clonality will be influenced by the platform, sample type, copy-number state, DNA quality, data quality, and size of imbalance. A discussion about the detection of mosaicism and acquired clonality by CMA is outlined in more detail later in these technical standards.

- The mechanism of some genetic imbalances (e.g., tandem duplication versus unbalanced insertion versus marker chromosome), which may necessitate the use of conventional cytogenetic and/or FISH studies.

- Tetraploidy or other ploidy levels; although, CMA platforms incorporating SNP probes may facilitate detection of these abnormalities.

- Copy-number changes of genomic regions not represented on the CMA platform.

- Duplications and deletions below the detection level according to probe coverage and performance, single-nucleotide variants (SNVs) or insertions/deletions (indels) not covered by the platform, gene expression, or epigenetic modifications.

- All variants associated with a given disorder. Therefore, it must be understood that failure to detect a copy-number change at any locus does not exclude the diagnosis of a disorder associated with that locus.

- All significant clonal and subclonal cell populations; although, clonal diversity can be characterized.

Because of these limitations in a neoplastic setting, results using CMA technologies at diagnosis may need to be correlated with other established methodologies (G-banded chromosome and/or FISH analyses) whenever it is warranted.

\section{CMA platform design and manufacture}

CMA platforms currently available for clinical testing use oligonucleotide-based DNA probes. The oligonucleotide-based DNA probes may be designed to detect only copy-number changes of a sequence as compared with a control or may also be able to determine a specific genotype (or allele) associated with the probe (a SNP-detecting probe). The copy-number of a probe may be determined either through a directly competitive hybridization of differentially labeled patient and control DNA (i.e., array comparative genomic hybridization [aCGH]) or comparison of the intensity of the labeled patient DNA to an in silico reference set (i.e., SNP array). The copy-number data are plotted as a log2 ratio of the probe intensities, with the expected normalized value equaling " 0 " (generally associated with two copies of genomic sequence), relative DNA gains having signals of greater intensity $(\log 2>0)$, and relative DNA losses having less intensity $(\log 2<0)$. For SNP array platforms, the copy-number changes should also correlate with the allelic information assuming sufficient coverage of the copy-number changes with SNPdetecting probes. For example, a region present in one copy should only have single SNP alleles identified in the region. 
CMA platform designs may have probes (1) targeted to specific regions of the genome for detection of imbalances known to be associated with the disease of interest, (2) distributed in a genome-wide manner with a specified distribution and spacing, or (3) placed in both a targeted and genome-wide manner with varying distribution and spacing of probes for specific genomic regions as well as across the genome. The functional resolution of a CMA will be determined by both the intermarker probe spacing and the number of consecutive probes necessary to confidently identify a true copy-number change. The functional resolution may be different across different regions of the genome for a given platform due to probe density and may vary for copynumber gains and losses as reflected by the log 2 ratio.

Manufacturers of CMA platforms should verify the identity of each probe on the platform used for clinical testing. Probes selected from the public domain should be listed with their physical and cytogenetic positions on the human genome, including the genome build. All probe descriptions and annotations should be openly accessible to the performing laboratory. Details regarding the CMA design, the synthesis verification, and all quality control (QC) steps taken to validate and assess the performance and reproducibility of the CMA should be documented and provided by the manufacturer. Additional information may be found in the ACMG recommendations for the design and performance expectations for clinical genomic copy-number microarray devices. ${ }^{15}$

CMA should be designed with consideration of the statistical algorithms to be used for determining abnormal thresholds. The number and density of probes within a given region of interest (i.e., within a region known to be associated with a germline disorder or cancer gene or feature) should provide the sensitivity needed for detection of a copy-number change.

\section{METHODS}

These technical laboratory standards were informed by a review of the literature and current guidelines. Resources consulted included PubMed; relevant ACMG, ACOG, and SMFM guidelines; and current World Health Organization (WHO) guidelines. The workgroup members also used their expert opinion and empirical data to inform their recommendations. Any conflicts of interests for workgroup members are listed at the end of the paper. The ACMG Laboratory Quality Assurance Committee reviewed the document providing further input on the content, and a final draft was presented to the ACMG Board of Directors for review and approval to post on the ACMG website for member comment. Upon posting to the ACMG website, an email and link were sent to all ACMG members inviting participation in the 30-day open comment process. All members' comments and additional evidence received were assessed by the authors, and these recommendations were incorporated into the document as deemed appropriate. Member comments and author responses were reviewed by representatives of the ACMG Laboratory Quality Assurance Committee and the ACMG Board of Directors. The final document was approved for publication by the ACMG Board of Directors.

\section{FAMILIARIZATION WITH A NEW TECHNOLOGY FOR THE LABORATORY BEFORE VALIDATION}

The laboratory with little or no experience with CMA technology should become familiar with all aspects of the new technology before beginning the validation process, regardless of the regulatory status of the array. Familiarization begins with understanding of the processes, features, and capabilities of the technology selected. The laboratory should gain experience with the instrumentation, platform design, software, reagents, methodology, technological limitations, workflows, DNA quality parameters, etc., by experimental sample runs.

Similarly, the laboratory should become familiar with the features of each sample type the laboratory will process, as different sample types may have unique considerations for CMA data quality and clinical applicability. The laboratory should demonstrate expertise in technical performance of the CMA, reproducibility of results, and data analysis and interpretation. Expertise should be documented for each CMA platform used for clinical testing, regardless of whether the laboratory has prior experience with a different platform. The laboratory must also be familiar with the potential imbalances and rearrangements associated with the clinical indications.

It is strongly suggested that laboratories use data from wellcharacterized samples to gain and broaden their experience. Sample exchanges with a laboratory proficient with CMA technology can provide a good source of samples for validation. Exchange of validated data sets between laboratories provides additional experience in data analysis. Samples chosen for validation studies should have aberrations that challenge the technical limits of detection for reportable deletions and duplications.

Laboratories need to be able to recognize nonperforming (or nonresponsive) probes, technically induced artifacts, and other issues affecting data quality. Laboratories should become familiar with CNVs that are benign and/or common and resources to aid in the recognition and interpretation of CNVs or CNAs. ${ }^{15-21}$

\section{VERIFICATION AND VALIDATION}

\section{Definitions}

Verification. Verification is a confirmation, through provision of objective evidence, that specified requirements have been fulfilled. This is a one-time process completed to determine or confirm test performance characteristics before the test system is used for patient testing. Verification is a quality assurance (QA) process to determine that instruments, software, and associated data are accurate per the manufacturer's description and specifications, i.e., does the system (hardware, software, probes) function as described by the vendor/manufacturer? Verification is required when using Food and Drug Administration (FDA)-cleared/approved tests. For the purposes of these technical standards, the terms "FDA-cleared tests" and "FDA-approved tests" will be interchangeably used and denoted as "FDA-cleared/ approved tests."

Validation. Validation is a confirmation, through the provision of objective evidence, that requirements for a specific intended use or application have been fulfilled. Validation is a QC process to determine that the data from test samples are accurate for the intended use when compared with a validated method, i.e., does the system (processes) provide the correct (accurate, reproducible) result(s) when test samples or test data are analyzed? Validation is required when using laboratory-developed tests or modified FDA tests.

New platform. A new platform is defined as any new methodology or microarray type introduced into the laboratory. A single microarray vendor may produce multiple similar platforms, but each must be assessed independently.

New version. The definition of a new version should be limited to those situations in which a minimal number of probes are removed, added, and/or replaced for the purpose of improved performance, and/or coverage is enhanced over a limited number of genomic regions. This would likely involve $<10 \%$ of the total probe coverage, with no more than $5 \%$ probe removal. It should be recognized that these types of changes to an established platform are likely a rare event and most changes in platforms will require a full validation.

All platforms intended for clinical testing must be either FDAcleared/approved and verified or must be validated by the 
performing laboratory. The extent of work necessary for a validation can depend in part on whether the laboratory is validating a new microarray platform for the laboratory, validating a modified design of a previously validated version, or adding additional sample types or intended uses to a previously validated platform. The scope of the verification, and method and scope of the validation must be documented.

\section{Verification of an FDA-cleared/approved test}

For any FDA-cleared/approved microarrays where the laboratory plans to claim the test as FDA-cleared/approved, the approved protocol and intended use (usually included in the package insert) must be followed. The laboratory must verify that it can obtain comparable performance specifications as those established by the manufacturer with regards to accuracy, precision, and reportable range of results. Any modification to the FDAcleared/approved use of the product (as specified in the package insert) will be considered as off-label use, and therefore the microarray must then be validated as a non-FDA-cleared/ approved platform.

At the onset of verification, pass/fail criteria for the verification protocol should be established. Each laboratory should define the pass/fail criteria for quality control metrics at various steps of the assay. If the prespecified acceptance criteria are not met, and a repeat or evaluation of the reasons for the failure does not resolve the concern, the laboratory should consider whether or not the array is appropriate for clinical testing.

Accuracy testing will measure the ability of the platform and software to detect known abnormalities. The accuracy evaluation is accomplished by running a series of previously characterized abnormal samples (this may be accomplished through sharing samples with an established laboratory). A minimum of 15 cases is recommended. To the extent possible, the laboratory should use abnormal samples that represent abnormalities that the array is designed to detect. This evaluation should include both a comparison of the findings from the region(s) expected to be abnormal as well as a comparison of the rest of the genome analyzed by the platform. The laboratory must document the concordance of the expected results and any unexpected findings. Because this technology may detect true alterations not previously identified, any unexpected findings that fall within the determined reportable range (as defined in the "Validation of a new CMA test for the laboratory" section) should be further investigated to determine whether the finding represents true biological variation. This may involve the use of an alternative technology, e.g., multiplex ligation-dependent probe amplification (MLPA), quantitative polymerase chain reaction ( $q P C R$ ), FISH, or a different microarray platform for correlation of the unexpected finding.

Precision testing should measure the reproducibility of repeated tests for the same result. The precision of the platform is established by running a minimum of two abnormal samples, each run multiple times in separate experiments. The concordance of the repeated runs should be documented, and any alterations should be considered (variability of breakpoints, calls, and potential reasons for variation, i.e., segmental duplication-rich region) as they pertain to the reportable range, functional resolution, and potential variability around breakpoints. Some variability around breakpoints may be expected due to segmental duplications and individual probe performance. Precision testing can allow for an assessment of breakpoints and potential impact on the clinical interpretation. Breakpoint variability that does not alter the clinical interpretation would be less concerning than variability that does alter the interpretation. Samples with multiple abnormalities are preferable as they maximize the number of findings for the precision study.
Validation of a non-FDA-cleared/approved test

Validation of a non-FDA-cleared/approved test is specific for each analysis type (constitutional, neoplastic, or formalin-fixed paraffinembedded [FFPE] tissue), which are considered different tests. At the onset of validation, pass/fail criteria for the validation protocol should be established. If the prespecified acceptance criteria were not met, and a repeat or evaluation of the reasons for the failure does not resolve the concern, the laboratory should consider whether or not the array is appropriate for clinical testing.

\section{Validation of a new CMA test for the laboratory}

Validation of a new CMA test includes establishing the performance characteristics of the microarray platform and software, in addition to technical data analysis and interpretation. The performance characteristics that must be established include the accuracy and precision of results, the analytical sensitivity and specificity, and the reportable ranges. Validations should be documented for each new clinical CMA test, regardless of whether the laboratory has prior experience with a different platform.

The reportable range of results includes criteria to identify a copy-number change, and criteria to report it. Laboratories, with consideration of the manufacturer's recommendations, should identify the parameters specific to their platform (number of consecutive probes, log2 ratios, SNP allele ratios, QC metrics, etc.) that are necessary to conclude that a copy-number call represents a true copy-number change. As the functional resolution is a combination of probe density and number of probes necessary to identify a true copy-number change, the reportable range should be at or above the functional resolution of the platform. The reportable range should be determined before the evaluation of the validation set, and data from the familiarization process should be utilized. The reportable range may exclude well-characterized benign CNVs. If the reportable range is altered by the laboratory, the validation data should be re-evaluated with the new reportable range. However, if the previously identified validation samples do not contain abnormalities that challenge the altered reportable range, additional samples should be evaluated.

The accuracy evaluation is accomplished by running a minimum of 30 previously characterized abnormal samples. To the extent possible, the laboratory should use abnormal samples that represent abnormalities that the array is designed to detect. This should include both autosomal and sex chromosome abnormalities as duplications and deletions on the sex chromosomes may behave differently in each sex. Furthermore, blinding the evaluators to the expected abnormalities has the additional benefit of validating the settings, evaluation of data, and reportable range. Samples used for validation should represent a variety of positive results with various sizes of abnormalities, combinations of gains and losses, various regions of the genome, and some aberrations that challenge the technical limits of detection for reportable DNA copy-number gains and losses.

Sample exchanges in a blind, split-sample comparison with a laboratory that is proficient with microarray technology can provide a good source of samples for validation. This sample exchange should include abnormal samples and involve comparison of results at the appropriate detection levels declared by the laboratories. Exchange of validated data sets (e.g., array files) between laboratories is recommended for additional experience in data analysis. All validation data for multiple disease and sample types, including discordant results and limitations, should be documented.

This evaluation should initially include a full review of the data to identify aberrations that meet the reportable range while blinded to the expected abnormality (as would fit the clinical workflow), followed by a comparison of the findings from the region(s) expected to be abnormal, as well as an evaluation of the rest of the genome analyzed by the platform. An evaluation of the regions expected to be normal is also important in assessing 
the probe behavior across the genome. The laboratory must document the concordance of the expected results and any unexpected findings. Sample assays for a specific diagnosis may be validated by comparison of results with those obtained by other methods, e.g., conventional cytogenetics, FISH, or another validated microarray assay. During the validation process, all genomic imbalances identified by standard method(s) should be detected by the microarray within the detection limits established by the laboratory for the diagnosis and/or sample type. Evaluation should also include breakpoint assessment with regard to gene content and genomic architecture. The laboratory should also recognize nonresponsive probes in a region expected to show loss or gain (this may be due to either poor performing probes or underlying genomic architecture). As this technology may detect true alterations not previously identified, any unexpected copynumber changes that fall within the laboratory-determined reportable range should be further investigated to determine whether the finding represents true biological variation. This may involve the use of an alternative technology, e.g., MLPA, qPCR, $\mathrm{FISH}$, or a different microarray platform for correlation of the unexpected finding. As both expected and unexpected findings are evaluated, careful selection of the 30 samples is important and the ability to evaluate unexpected findings should be considered.

Sensitivity and specificity are determined by the number of true positive, true negative, false positive, and false negative results in a validation data set that meet reporting criteria.

However, for a whole-genome assay, all true positives and true negatives are not known. Therefore, sensitivity and specificity for genome-wide array tests cannot be calculated as traditionally defined.

Sensitivity is evaluated by comparison of expected versus observed abnormalities, and this is then extrapolated to the rest of the genome. Rather than a traditional calculation of specificity, an evaluation of the positive predictive value of the assay is desirable. Determination of the positive predictive value will involve the identification of copy-number calls that fall within the laboratory's determined reportable range and a determination of the proportion of those calls that are true. To improve the specificity of the platform, if certain probes are recognized to repeatedly act as false positives, these probes should be removed from future analyses. The identification of false positive probes may be due to technical or biological variables considering that not all regions of the genome are amenable to accurate locus-specific evaluation of copy-number with this technology. If probe content is masked by the laboratory, these changes should be documented. If the changes are sufficient to alter the performance of the platform, an evaluation of the validation data with the altered probe content is required.

The precision testing should measure the closeness of repeated test results to one another. The precision of the platform is established by running a minimum of two abnormal samples, each run multiple times in separate experiments. The concordance of the repeated runs should be documented, and any alterations should be considered (variability of breakpoints, calls, and potential reasons for variation, i.e., segmental duplication-rich region) as they pertain to the reportable range, functional resolution, and potential variability around breakpoints. Some variability around breakpoints may be expected due to genomic architecture and individual probe performance. The precision testing can allow for an assessment of breakpoints and potential impact on the clinical interpretation. Breakpoint variability that does not alter the clinical interpretation would be less concerning than variability that does alter the interpretation. Samples with multiple abnormalities are preferable as they maximize the number of findings for the precision study.

2. Validation of a new version of a previously established platform In the laboratory that is proficient with microarray technologies, a new version of a platform in use by the laboratory from the same manufacturer should be validated with a minimum of five abnormal samples. Known abnormal samples from the previous version should be run using the new version for comparison to ensure that the performance meets the laboratory standards and to assess performance of probes added into a higher-resolution version. New content on an upgraded version should be assessed, if possible, using known abnormal sample(s) with variation in the region of the new content to determine performance.

The evaluation of this validation set of at least five samples should include data analyzed to determine whether the platform and software detected the expected abnormality. If other abnormalities are detected that meet the laboratory-reporting range, the validation should determine whether the findings represent true biological variation.

3. Validation of additional sample/tumor types on an established platform

It is understood that the CMA platform employed by the laboratory may be used to analyze multiple sample types and, in a neoplastic setting, multiple neoplastic disorders. It is expected that the initial validation will involve the most common sample type for the expected intended use. For example, if the intended use is postnatal constitutional evaluation, the sample type will likely be DNA extracted from peripheral blood, whereas if the intended use is neoplastic hematologic malignancy evaluation, the sample type will likely be DNA extracted from bone marrow or peripheral blood.

Because the quality of the DNA may vary from alternative tissue/tumor sources and this may add interference factors to the CMA analysis, use of DNA from alternative sample types requires an evaluation of the potential for interference. ${ }^{22}$ Inherent differences in results obtained from different biological materials require that the laboratory determines the performance characteristics of the CMA for each sample type to be used for clinical testing. Examples include constitutional or neoplastic blood, neoplastic bone marrow, fresh or frozen tissue/tumor, and FFPE tumor.

For a new sample type, an evaluation of the impact of the new sample type on data quality is necessary. The DNA extraction process should be part of the validation plan. Evaluation of the array QC metrics of the new sample type is critical to ensure that they are within the established acceptable range. If there are only minimal changes to the processing or analysis, then a validation of the new sample type can involve equivalency of data quality with the new sample type. If significant alterations are made in the processing of the sample or CMA analysis (e.g., change of reference DNA), then a new validation is required.

In a neoplastic setting, laboratories often offer clinical testing for different neoplastic disorders using different sample types. In this case, the laboratory should process and analyze a sufficient number of each type to establish proficiency. Tumor-specific sample types for which clinical testing will be offered should be included in the validation. Each laboratory should use professional judgment and experience to determine the number of samples of a particular neoplastic disorder to include in their preclinical testing validation. Laboratories will also need to use professional judgment and experience to determine differences in processing various sample types and adjust sample numbers of each type accordingly, with the goal of optimizing quality and analytic interpretation of results.

4. Validation of the allelic differentiation potential of SNPdetecting platforms

In postnatal constitutional CMA, the detection of $\mathrm{ROH}$ is not in and of itself diagnostic but can identify a concern that may require additional testing such as sequence-based variant analysis or uniparental disomy testing. In the neoplastic setting, the detection of CN-LOH with or without additional CNAs may be diagnostic of 
certain types of malignancy, and/or have therapeutic or prognostic implications. Given sufficient probe density, there should be a correlation between the copy-number state and the SNP allele state. Evaluation of the performance of the SNP-detecting probes to define $\mathrm{ROH}$ or $\mathrm{CN}-\mathrm{LOH}$ should be included in the validation.

A minimum of five samples need to contain expected $\mathrm{ROH}$ or $\mathrm{CN}-\mathrm{LOH}$ in addition to copy-number changes. Interlaboratory comparisons of such samples are recommended. This comparison should address the data types that would be included in a report, such as approximate sizes of $\mathrm{ROH}$ or $\mathrm{CN}-\mathrm{LOH}$, and percentage of the genome demonstrating $\mathrm{ROH}$ or $\mathrm{CN}-\mathrm{LOH}$, whenever clinically applicable. The detection and accurate size assessment of $\mathrm{ROH}$ or CN-LOH by SNP-based CMA depend on the density of SNP probes. If the validation method does not address accuracy of breakpoints in $\mathrm{ROH}$ or $\mathrm{CN}-\mathrm{LOH}$ calls, reports should reflect this uncertainty in the disclaimer section. In constitutional CMA, inaccurate size or breakpoint estimation for $\mathrm{ROH}$ may lead to unwarranted follow-up testing for uniparental isodisomy and/or autozygosity mapping, ${ }^{23}$ and in neoplastic CMA, inaccurate size or breakpoint estimation for CN-LOH may lead to misinterpretation of a variant as homozygous when a somatic variant is detected in a suspected region of $\mathrm{CN}-\mathrm{LOH}$.

5. Determining percentage of cells with abnormality: mosaicism and clonality

Constitutional mosaicism, the admixture of non-neoplastic cells in a tumor sample and clonal diversity can readily be detected by CMA. However, each laboratory needs to perform extensive validation studies to determine the dynamic range and the limit of detection for different cell populations for a wide variety of copynumber changes. For constitutional studies, it is not recommended that this technology be used as the sole method to rule out mosaicism. In cases with apparent low percentages of cells with questionable aberrations, FISH, conventional cytogenetics, or another quantitative method may be needed to fully characterize the genetic lesion(s).

The ability to detect mosaicism or clonal changes can be influenced by several factors including the microarray platform used, sample source, DNA quality and quantity, size and copynumber state of the abnormality, and probe coverage. Noise from poor-quality DNA and mixed chimerism may mask clonal abnormalities. Each laboratory will need to challenge their microarray with various percentages of abnormal cells, different ploidy levels, and clonally diverse samples to gain experience in their detection. It is not likely that a specific percentage of cells with an aberration will always be identified uniformly throughout the genome. This could be due to either poor performing probes, and/or genomic content affecting microarray performance, and this limitation should be recognized.

Methods for determining detectable percentages of cells include dilution series studies from an admixture of normal and abnormal cells from the same individual (if possible), obtaining samples from another laboratory with known abnormal cell percentages, and analysis of the mosaic sample by other quantitative methods. FISH analysis of fresh (uncultured) samples provides a reliable means to establish the percentage of cells with an aberration to compare with the microarray data. Flow cytometric data may also be used to estimate clone size (e.g., blast percentage for acute leukemia). Conventional cytogenetic analysis of metaphase cells provides information about mosaicism but may not accurately reflect levels of mosaicism. Note that methods to evaluate levels of detectable mosaicism/clonality will differ with sample type, e.g., fresh or FFPE tissue.

Dilution studies using samples with known copy-number changes may help to determine detectable levels of mosaicism. ${ }^{24}$ This method can provide an effective means of establishing thresholds but may have limitations as a simulated method. For SNP-detecting arrays, dilution studies require non-neoplastic and tumor DNA from the same patient; buccal cells or blood may provide a source of non-neoplastic patient DNA. Be aware that microarray analysis gives a relative level of copy number across the cells within the sample but does not provide a cell-by-cell determination of copy number (e.g., trisomy in $60 \%$ vs. tetrasomy in $30 \%$ of cells).

Microarray analysis tools were designed primarily for nonmosaic abnormalities; therefore mosaicism/clonality may not be reliably detected by the standard software algorithms. Laboratories should recognize software limitations and the need for manual and visual inspection of the data for mosaic aberration and clone/subclone detection. For validation, clinically relevant calls made by visual/ manual inspection and calls made by software should be verified by another method, e.g., interphase FISH, qPCR, and/or replicate array analysis.

The percentage of cells with a specific abnormality in a mosaic or clonal state can be estimated using software parameters, including the log2 ratio, B-allele frequency, and/or allele difference. ${ }^{24-27}$ However, the estimate is influenced by factors such as array platform, type of mosaic abnormality (i.e., one-copy loss or gain, two-copy loss or gain, and ploidy changes), and array quality. For example, the log 2 ratio of the same mosaic percentage may be different between aCGH and SNP array. Each laboratory should consider these variables and be familiar with the capabilities/limitations of the array analysis software used.

\section{Special considerations}

6.1. Special considerations for validation of prenatal specimens

Experience with postnatal CMA and with common and rare CNVs is important for the processing and interpretation of array results in the prenatal setting. For validation, a distinction should be made between cultured amniotic fluid and chorionic villus sampling (CVS) cells and uncultured (direct) amniotic fluid and CVS cells. The validation depends on whether the platform has been previously validated for postnatal use or is new to the laboratory and whether both cultured and uncultured cells will be used. Both cultured and uncultured amniotic fluid and CVS should be included in the test validation, especially if the laboratory plans to perform CMA analysis on all these sample types.

Analysis of DNA extracted from uncultured amniotic fluid or CVS cells is preferable to DNA from cultured amniotic fluid or CVS cells. CMA analysis of uncultured amniotic fluid and CVS cells allows for the great majority of results to be available within one week and avoids the possibility of culture artifacts. ${ }^{28-31}$ Maternal cell contamination (MCC) is a possibility when studying uncultured amniotic fluid or CVS cells (see MCC recommendations below). The villi need to be manually cleaned of maternal decidua prior to DNA extraction. Confined placental mosaicism (CPM) is a concern when studying uncultured CVS cells, but only a low frequency of CPM in CMA analysis of uncultured CVS cells has been reported. ${ }^{32}$ This could be attributed to the evidence demonstrating that cleaned villi are mostly composed of the mesenchymal core, which is more representative of the fetal genome. ${ }^{33}$ Back-up cultures for all prenatal samples undergoing CMA analysis should be established and maintained. This is necessary for the purposes of (1) possible array failures using direct DNA extractions, (2) evaluation of possible mosaicism, and (3) the need to perform metaphase chromosome or FISH analysis to investigate CNVs.

If prenatal CMA is performed on an array platform new to the laboratory, the issues and process discussed in the "Validation of a new CMA test for the laboratory" section apply, and a minimum of 30 previously characterized cases should be processed. Due to the difficulty of obtaining abnormal prenatal specimens, the collection of 30 samples will likely include those cases previously characterized as normal. Additional experience with abnormal array findings through data exchange should occur, to ensure that a wide variety of abnormalities have been evaluated both in-house and in silico. 
For a previously validated platform for postnatal use, the addition of prenatal specimens requires an understanding of the potential issues that these samples can present regarding DNA quantity and data quality. The DNA extraction process should be part of the validation process. A minimum of five samples from each sample type, uncultured amniotic fluid, uncultured CVS, or cultured amniotic fluid/CVS, should be included in the preclinical testing validation.

Uncultured prenatal samples. Because uncultured cells may yield inconsistent DNA quantity and quality, additional validation may be required to become familiar with potential differences as compared with cultured cells. Parameters to consider for uncultured amniocytes include amniotic fluid volume, gestational age, and DNA extraction method. In general, uncultured amniocytes yield less DNA than cultured cells; however, CMA results are obtained faster.

Cultured prenatal samples. Healthy cultures established from amniocytes, CVS, and fetal tissue need to yield an adequate quantity and quality of DNA and provide consistent CMA results. The laboratory needs to be aware of factors that can affect DNA yield and data quality such as culture age, growth rate, confluency, and shipping conditions.

Maternal cell contamination and mosaicism. It is recommended that prenatal samples submitted for CMA be assessed for MCC. MCC may be present in direct samples of amniocytes that contain maternal blood, in CVS samples not adequately cleaned of maternal decidua, and in cell cultures after extensive subculturing with maternal cell expansion. Underlying MCC may affect detection and interpretation of CNVs, including different CNV types (gains and losses) and different CNV sizes (small versus large gains and losses). Low-level mosaicism in fetuses may be missed in the face of a significant level of MCC.

MCC may be detected with different methods including short tandem repeats (STR) analyses and SNP-based CMA platforms. For male fetuses, a shift in the sex chromosome plots mimicking mosaicism can suggest MCC. Each laboratory should validate their method for MCC detection to discern the level of MCC that is acceptable for their particular CMA platform. The acceptable level of MCC in uncultured samples should be determined to assess when cultured cells would be best for obtaining a successful CMA analysis.

Mosaicism detected by CMA should be investigated to confirm its presence and level and may represent a culture artifact (pseudomosaicism), true fetal mosaicism, or for CVS, CPM. ${ }^{32}$

Coverslip colony cultures may be used to investigate mosaicism (see section E4 of the ACMG Technical Laboratory Standards). Depending on the chromosome involved and the type of abnormality, additional studies using a different sample (e.g., amniotic fluid in a CVS sample suspected for CPM) may be considered to confirm or exclude mosaic status. FISH analysis may be used to investigate presence and level of mosaicism but may be unable to distinguish true from pseudomosaicism.

6.2. Special considerations for validation of oncology specimens

The validation of oncology specimens will follow the "Validation of a new CMA test for the laboratory" section regardless if the laboratory has experience with postnatal CMA, and a minimum of 30 previously characterized cases should be processed. Experience with postnatal CMA and with common and rare CNVs is helpful for the processing and interpretation of array results for oncology specimens. If multiple tumor types from different tissue sources will be processed using CMA, the DNA extraction process should be validated following the "Validation of additional sample/tumor types on an established platform" section.

Clonal diversity. CMA uses an averaged DNA pool of all cells in the sample; thus, clonal and subclonal populations may not be accurately defined from CMA data. However, combinations of multiple abnormalities with the same estimates of cell percentages can be used to infer information about clones, including delineating clonal diversity of the tumor. Clonal diversity, common to neoplastic disorders, is observed when the cell populations of different clones reach the threshold for detection. Additionally, independent clonal populations can occur in the same tumor tissue and CMA alone will not be able to distinguish unrelated clones or subclones. Correlation with conventional cytogenetic and FISH analyses can aid in the interpretation of the tumor clonal and subclonal composition. CMA platforms in use or in the process of being validated should be assessed for the capability of detecting clinically significant CNAs and CN-LOH (i.e., tiers 1 and 2) within clonally diverse cell populations. $^{12}$

Germline abnormalities. CMA testing in neoplasia may uncover germline abnormalities in patients. The interpretation and reporting of unanticipated clinically significant germline variants should be performed in accordance with "Technical laboratory standards for interpretation and reporting of acquired copynumber abnormalities and copy-neutral loss of heterozygosity in neoplastic disorders"12 and "Points to consider for reporting of germline variation in patients undergoing tumor testing." ${ }^{134}$

\subsection{Determination of ploidy}

Hypodiploidy, hyperdiploidy, and polyploidy can be detected by CMA but may be challenging to appreciate and interpret. The allelic states of SNP probes can assist in determining ploidy levels; allele differences and/or B-allele frequency and log2 ratio together assist in determining the diploid baseline. The validation process should include samples with varying levels of ploidy to gain experience in analysis and recognition of different ploidies. Correlation with FISH and karyotype can help to determine the ploidy level and the potential need to readjust the diploid baseline. If FISH and karyotype are not available, the triallelic region (balanced genotypes with homozygous $A$ and $B$ and heterozygous $A B$ alleles) with the lowest log2 ratio often represents the diploid baseline, and the rest of the genome may be normalized accordingly. It may be necessary to confirm that CNAs detected by this approach are reported as recurrent abnormalities in the neoplastic disorder under investigation. The manufacturer should provide the method used for normalization. The laboratory must understand the effect that normalization may have on polyploidy detection and subsequent interpretation of gains and losses in the context of polyploidy.

\subsection{Chimerism}

A mixture of genotypes may occur with constitutional samples (e.g., MCC in prenatal samples) or cancer samples (e.g., following stem cell/bone marrow transplantation) and can also be detected when clinical samples are inadvertently mixed. It is important to recognize the specific pattern that is generated from chimerism. When two samples are mixed together, the SNP allele tracks become increasingly complex. Thus, it may not be possible to determine the origin of the major and minor contributions without known copy-number changes or $\mathrm{ROH} / \mathrm{CN}-\mathrm{LOH}$ from at least one of the individuals (e.g., germline CNVs or previously reported patient abnormalities). Adjunct tests to assess the levels of chimerism, such as STR analysis, can assist with estimation of the contribution of individuals. When chimerism is present, the ability to detect low levels of cells with abnormalities and small aberrations will be impacted and data interpretation may be compromised. In addition, the report should indicate the limited analysis, given the complexity of the results from chimerism.

\section{REFERENCE SET CONSIDERATIONS}

Depending on the array platform used, the reference set may come from a single individual or multiple individuals, may be sex matched or mismatched, and may be used in silico (with SNP- 
based microarray) or as a direct competitive hybridization (with $\mathrm{aCGH}$ ). The laboratory should understand the benefits and limitations of each scenario. The laboratory should be aware of how the data quality may be affected by the source and components of the reference set. For example, data quality is likely improved when the assay conditions used for the reference set closely match those for the test. ${ }^{35}$ Any changes to the reference set could alter the results, and therefore require a verification of the quality and accuracy of results obtained with the new set as compared with the previous one.

\section{Array comparative genomic hybridization analysis}

aCGH analysis requires comparison of sample DNA to reference (control) DNA in the assay, thus the selection of an appropriate reference DNA is essential. Laboratories may establish their own reference DNA or use reference DNA provided by the manufacturer. The laboratory should characterize any reference DNA to identify germline CNVs that may have an effect on the interpretation of patient data. During the familiarization phase, it is important to optimize the reference DNA to ensure high quality data. This includes the DNA extraction process, purity, and concentration. Fluorometric quantitation of the DNA is recommended to ensure that equal quantities of sample and reference DNA are used in the assay.

Laboratory policies should detail how reference DNA will be used, i.e., mismatched opposite-sex or same-sex comparisons, as single male or single female references, or as pools from multiple male or multiple female DNA samples. The laboratory should document the rationale for the use of reference DNA types and have provisions for use in different situations. The advantages and limitations of different approaches should be understood and considered during interpretation of data. The QC metrics of each new lot of purchased reference DNA should be compared with the previous lot to ensure that they are within the expected range and to verify the accuracy and reproducibility of results.

In an oncology setting, constitutional DNA from blood or normal tissue from the same individual may be used as the reference DNA. While constitutional patient DNA will mask germline CNVs and reduce the complexity of postanalytic interpretation, novel underlying germline abnormalities that could contribute to disease will not be detected.

\section{SNP-based microarray analysis}

SNP-based microarray analysis requires comparison of the sample result with established references or an in silico reference library. If sufficient data are available for a control population, a laboratory may establish an in silico reference that mimics the typical study population. In creating the internal reference file (sometimes referred to as the "cluster" file), the laboratory minimizes interlaboratory variation resulting from varied equipment and other external conditions, and normalizes the data to minimize population variation. The laboratory should also consider variables that may differ between reference sample and test sample, such as DNA extraction methodology and sample type (e.g., fresh tissue versus FFPE sample). The laboratory should follow the manufacturer's recommendations for the minimum number of male and female controls used by the analysis software.

Laboratory policies should document the rationale for the use of an internal reference file and detail how reference files will be used. Reference files may be updated by adding, removing, or replacing samples. A new reference file should be established for new SNP-based array designs.

\section{SOFTWARE CONSIDERATIONS}

The laboratory should recognize software limitations and the need for visual inspection of the data. Manual calls are often necessary during validation and clinical testing, for example, to combine calls that are interrupted by poor performing probes, to separate calls that are interrupted by a normal region, to add calls for lowlevel mosaic aberrations that are not flagged by software, or to revise breakpoints not assigned accurately. To verify that the method for result generation (including software and manual calls) detects known aberrations accurately, the laboratory should test a variety of copy-number changes (i.e., deletions, duplications, and amplifications), $\mathrm{CN}-\mathrm{LOH}, \mathrm{ROH}$, and aberrations at different mosaicism/clonality levels. During the familiarization phase, the software settings should be optimized for aberration detection and then established parameters should be used consistently throughout the validation process. These include thresholds for size/number of probes, $\log 2$ ratio thresholds, and mathematical algorithms used by the software to make calls. The software parameter settings may be different for various sample types.

The laboratory must determine and document the ability and accuracy of the software to detect copy-number changes according to software rules and parameters. When applicable, the laboratory should determine the ability of the software to accurately define the endpoints of $\mathrm{CN}-\mathrm{LOH}$ and $\mathrm{ROH}$ according to the software settings within the resolution of the array design. Limits should be verified whenever the microarray platform, version, software, or analysis rules change. The laboratory should challenge the software with copy-number changes that help define the limits of detection.

Changes to the software settings from those used during the validation may require a reanalysis of at least a subset of the validation data using the new settings to identify any changes to the performance characteristics of the microarray platform. Such changes may include, but are not limited to, new annotation libraries, changes to any in silico reference set, or any changes to the aberration-calling algorithm.

The laboratory should understand that most normalization algorithms assume a primarily diploid state, which may obscure the detection of polyploidy. The allelic states of SNP probes may assist with the detection of hypodiploidy, hyperdiploidy, and polyploidy. These situations are rare in the postnatal constitutional samples but are relatively common in products of conception and oncology samples.

Any upgrade to the software that offers a change or improvement over previous versions is deemed a new version of the software. Laboratories should validate a new version of the software from the same manufacturer with a minimum of five abnormal samples generally representative of the different types of aberrations that arise in clinical testing. Known abnormal samples from the previous version should be analyzed using the new version of software to confirm that the new version detects the expected abnormality. If other abnormalities are detected that meet the laboratory-reporting criteria, the laboratory should determine whether the findings represent true biological variation. A new function in an upgraded version should be assessed, if possible, using known abnormal sample(s) with abnormalities that can test the new function and determine its performance. For example, samples with mosaic triploidy can be used to evaluate the new function of ploidy adjustment.

The laboratory should document the software parameters and rules used for the microarray analysis, and all limitations of the analysis program. The limits, rules, and parameters for detection of mosaicism/clonality should be determined.

\section{QUALITY CONTROL}

\section{Identification}

For each microarray, the slide identification number, subarray position (when applicable), sample sex, control sex (when appropriate), and sample-tracking control (for multiplex 
1826

microarrays) should be verified and a system developed to ensure sample identification throughout the process. Discrepancies in the documentation from the physical sample should be investigated and resolved before processing.

\section{Sample and DNA requirements}

The laboratory should establish sample adequacy requirements and parameters for the minimum DNA quality and quantity requirements for each sample type used for clinical testing. The laboratory should demonstrate proficiency in sample preparation, DNA extraction, and DNA purification for each sample type. Blood samples that do not meet the laboratory requirements can be rejected with a repeat sample requested from the referring physician. For prenatal and oncology samples where a repeat sample may not be available, the laboratory should attempt DNA re-extraction and/or purification and perform the array assay. Improvement approaches may include increasing the DNA input in the assay for certain sample types (e.g., FFPE samples) or wholegenome amplification, provided that the laboratory has expertise with this method and potential biases inherent in the technique are detailed in the report. Laboratory policies and protocols should describe when and how whole-genome amplification is performed.

A surgical pathologist should evaluate FFPE samples to assess tissue quality and select an area from the tissue block that contains ample (suggested minimum of $25 \%$ ) tumor or villi from products of conception to avoid masking copy-number changes in the tissue of interest by DNA from the normal tissue or maternal decidua, respectively.

DNA extraction, purification, measurement, and amplification with different sample types

DNA extraction methods should ensure the highest-quality DNA possible from the sample type(s) tested by the laboratory. FFPE samples present unique challenges for generating high quality DNA from the tissue of interest. Written protocols should be available in the laboratory procedure manual and/or quality management program for optimizing DNA extraction and labeling, DNA quantification (e.g., fluorometer, spectrophotometer), DNA quality and concentration, DNA fragmentation (e.g., via sonication or enzymatic digestion), fluorescent labeling (e.g., examination by gel electrophoresis, visual inspection, ultraviolet/visible spectroscopy), and amplification. For any labeling method, acceptable ranges should be determined for proper dye incorporation. Protocols for optimization, e.g., re-extraction, re-purification, tumor cell enrichment for hematological samples (cell sorting or magnetic bead enrichment), and/or microdissection for paraffin-embedded tissue, should be available as appropriate. Laboratories should be aware that fixatives other than formalin may influence DNA quality and that decalcification of bony tumors may adversely affect DNA quality.

Equipment calibration, maintenance, and quality control

Equipment, instrumentation and methodologies employed during the validation and use of microarray platforms should be calibrated, receive regular maintenance, and be monitored for QC. Quality metrics should be established for each step of the assay. Laboratories should ensure that data are processed and summarized in a consistent fashion for every clinical analysis. Most analysis software provides a hierarchy of users with customizable permissions, which enables the laboratory to prevent modification of analysis settings so that sample analysis is consistent. Any changes to data processing should be validated and documented.

\section{Quality control metrics}

Every microarray platform has defined quality metric values, e.g., adequate dye incorporation and/or amplification, fluorescence intensities variance, signal-to-background-noise ratio, and standard deviation or standard error. Standard cutoff values and acceptable limits should be established for these metrics to ensure that the generated results are reliable and sufficiently precise to be used for a clinical assessment. Quality metrics should be monitored for DNA labeling, hybridization efficiency, data generation and analysis, and other platform-specific parameters. QC metrics should be incorporated into the laboratory QA and quality improvement programs to monitor analytical variables.

\section{Microarray content}

It is not feasible for a laboratory to validate the identity and copynumber performance of every probe on a microarray. The laboratory should obtain documentation from the microarray manufacturer that the probes on each microarray are the intended sequences, located appropriately by the software, empirically selected for appropriate copy-number responsiveness and/or SNP allele specificity, and stable for these assessments from lot to lot.

\section{Data quality}

The quality of the data will affect the ability to detect genomic aberrations; thus, the laboratory needs to understand the withinarray metrics provided by the analysis software and how each metric reflects the quality of the data. One metric that provides a measurement of noise or random variance unrelated to genomic location in the data is the derivative log2 ratio. The derivative log2 ratio is the difference between the log 2 ratio values of consecutive probes. Similar metrics of variance exist for each platform. Data quality may be assessed using platform-specific parameters.

The laboratory should establish acceptable ranges for each QC metric chosen to assess data quality. The manufacturer often provides these ranges; however, the laboratory may want to modify these ranges based on their experience with the microarrays during the validation process. The ranges may differ for different sample types. The laboratory policies should describe the appropriate follow-up procedure, should the data fall outside of these established ranges.

\section{Annotation/databases}

An integral part of the data analysis is accessibility and use of private and public annotations/databases during the analysis process. Because these annotations are critical for interpretation, it is important that these tools are carefully constructed and applied by the laboratory or software manufacturer. Critical annotations should be versioned and updated regularly. The manufacturers should provide mechanism(s) for updates to these annotations. For all reportable calls, the genomic content may need to be verified by an independent database source (e.g., UCSC Genome Browser). Documentation of resources and databases accessed for interpretation is recommended.

Verification of new lots of microarrays and/or reagents

Verification should ensure that new lots of microarray slides and/ or reagents perform in the same manner as the previous lot. The laboratory should have documentation of the microarray slides manufacturing QC (e.g., oligo synthesis verification, accuracy of SNP calls, or other defined control parameters). A new lot of microarray slides should be tested to ensure equivalency, preferably using a patient specimen with an abnormal result that has been tested on a previous lot. New lots of reagents (e.g., new labeling kits and consumables) should have documented equivalency between runs. This may be accomplished by documenting 
that the QC metrics meet certain set parameters for the new lot of reagents.

\section{Confirmation of specific copy-number changes}

With proper technical performance and analytical validation, it should not be necessary for the performing laboratory to further confirm a copy-number change called with the laboratory-validated parameters, after the validation stage. Each laboratory should establish a threshold (number of probes and/or genomic size, as well as other QC metrics) for declaring what constitutes a reportable abnormality with their assay. Features to keep in mind when assessing copy-number changes are the appropriate $\log 2$ ratio difference between data, the presence of uniform contiguous probe behavior within and adjacent to call, sharp copy-number state transitions at breakpoint boundaries, supportive SNP allele states (when applicable), and evaluation of least processed log2 ratio data (e.g., weighted versus not weighted). Any call-specific quality score provided by the software may be considered.

Since it is desirable to maximize detection of aberrations involving clinically significant genes and of aberrations in mosaic form (which may not generate a robust copy-number call), it is acceptable and appropriate at the discretion of the performing laboratory to evaluate calls that do not meet the laboratoryvalidated parameters. These calls may be flagged for review and correlated with the patient's clinical indication, and when appropriate, should be confirmed by an independent methodology if reported.

\section{QUALITY ASSURANCE}

Laboratory accreditation and personnel qualifications

Laboratory personnel must have documentation of education, degrees, and certifications as appropriate for the level of testing, as well as training, competency assessments, and continuing education as required by appropriate regulatory bodies, e.g., College of American Pathologists (CAP), CLIA, and Center for Medicare and Medicaid Services (CMS). The testing laboratory must have CLIA certification and state certifications as required to provide clinical testing. CAP accreditation is strongly encouraged.

\section{Indications and ordering for microarray analysis of neoplastic} disorders

Microarray analysis of tumors should be limited to specimens that contain ample tumor. The sample should be accompanied by an appropriate indication for the test. Clinical testing may be limited to neoplastic disorders for which unbalanced genomic abnormalities and/or CN-LOH are well documented to have diagnostic, prognostic, and/or therapeutic implication(s). Microarray analysis may not be optimal for tumor surveillance or detection of minimal residual disease depending on the limit of detection of the aberration and the ability to use more sensitive methods for monitoring. When applicable, alternative methods should be recommended to monitor patient response to treatment and for residual disease detection (e.g., FISH and/or qPCR). A clonal abnormality identified and confirmed at diagnosis may be used for follow-up. The same method used for confirmation (e.g., qPCR or FISH) is recommended for use in follow-up studies.

Laboratories may facilitate appropriate ordering by providing a directive or disease-specific testing menu. The test requisition should provide sufficient clinical and/or pathological information for the laboratory to assess the appropriateness of the test order.

\section{Proficiency testing}

The laboratory should participate in proficiency testing (PT) for sample and tumor types that are included in the laboratory test menu by participating in an external PT program when available through an appropriately deemed organization (e.g., CAP). In addition, the laboratory may establish external PT of normal and abnormal specimens by the exchange of DNA, in a blinded manner, with another laboratory performing microarray testing.

The laboratory should also establish internal PT of normal and abnormal samples as part of the laboratory internal QA program and ongoing quality improvement program. Correlation between microarray results run in parallel on different microarray platforms or correlation of microarray results with conventional cytogenetic and/or FISH results may be sufficient to provide ongoing proficiency. PT should be performed according to the CLIA 1988 guidelines.

Documentation of participation and the performance results of internal and external PT must be retained by the laboratory and made available to all accreditation agency inspectors. Failure to achieve agreement on external or internal proficiency tests should be documented and followed by investigation of the discrepancy with resolution. If indicated, appropriate remediation should be undertaken.

\section{Turnaround time}

Laboratory policies should define acceptable standards for microarray analysis test prioritization and turnaround times. Turnaround time should be clinically appropriate so the results are available for patient care management decisions. It is suggested that $90 \%$ of cases should have a final written report by 21 calendar days.

Documentation of problems

A logbook, database, or sample processing form should be created and used to track problems that may occur throughout the processing of samples, from sample intake to final report (e.g., sample adequacy and/or errors). Data from the QC metrics program can provide information for oversight of all processes. Ongoing collection of sample or process variances allows patterns or trends to be recognized and promptly addressed.

\section{USE OF ALTERNATIVE TECHNOLOGIES FOR MECHANISM DETERMINATION}

Determination of the mechanism leading to the detected copynumber change may be considered on a case-by-case basis because this may lead to better determination of recurrence risk in constitutional studies and provide clinically useful information in neoplastic cases (e.g., confirmation of gene fusion). Some mechanisms can be identified through the combination of both the copy-number change(s) and recognition of the genomic location of the altered material, or the genomic structure surrounding the alteration. Examples include unbalanced translocations and insertions, iso- or isodicentric chromosomes, and ring or marker chromosomes. The appropriate alternative technology may depend on the size, type, and location of the identified copynumber change(s) and the likely mechanism of formation. Therefore, use of these alternative technologies should be considered as separate testing and should use validated technologies performed and interpreted by appropriately trained personnel.

\section{INTERPRETATION AND REPORTING}

For further guidance on interpretation and reporting, refer to the published "Technical standards for the interpretation and reporting of constitutional copy-number variants" 21 and "Technical laboratory standards for interpretation and reporting of acquired copy-number abnormalities and copy-neutral loss of heterozygosity in neoplastic disorders" ${ }^{12}$ as well as "Standards and 
guidelines for documenting suspected consanguinity as an incidental finding of genomic testing. ${ }^{136}$

\section{METHODOLOGY AND DISCLAIMERS}

All reports should include a brief description of the methodology, including platform specifics and reporting criteria. Disclaimers should be included as appropriate and required.

\section{Example: testing limitations}

Current microarray analysis technologies will detect only gains and losses of genomic regions. Therefore, a normal microarray result does not exclude single nucleotide variants (SNVs) or insertions/deletions (indels) not covered by the platform, gains and losses below the level of resolution of the platform, a balanced rearrangement, or epigenetic events. Additional testing may be appropriate for certain syndromes or conditions when the microarray analysis yields normal results.

\section{Alternative example}

This microarray platform will not detect truly balanced chromosomal rearrangements, single nucleotide variants (SNVs) or insertions/deletions (indels) not covered by the platform, or imbalances of regions not represented on the microarray, and may not detect mosaicism. Failure to detect an alteration at any locus does not exclude all anomalies at that locus.

Example: disclaimer for a non-FDA-approved microarray platform This test was developed and its performance characteristics determined by (your laboratory name here) as required by the Clinical Laboratory Improvement Act (CLIA) 1988 regulations. It has not been cleared or approved for specific uses by the US Food and Drug Administration. Pursuant to the 1988 CLIA requirements, this laboratory has established and verified the test's accuracy and precision.

\section{RETENTION OF FILES AND DOCUMENTATION}

Laboratories should be explicit in their policies as to which file types and for what length of time each type will be retained and that data retention policy must be in accordance with local, state, and federal requirements. CLIA regulations (section 493.1105) require storage of analytic systems records and test reports for at least two years. For more specific suggestions for microarray technologies, we recommend that the laboratory consider a minimum of 2-year storage of a file type that would allow regeneration of the primary results as well as reanalysis with improved analytic pipelines. In addition, laboratories should consider retention of the aberrations identified in the analysis, along with the final clinical test report interpreting the subset of clinically relevant variants, for as long as possible, given the likelihood of a future request for reinterpretation of variant significance.

\section{CONCLUSIONS}

CMA technologies provide a high-resolution copy-number view of the whole genome. The clinical application of this technology for constitutional and neoplastic disorders requires extensive clinical validation to ensure the results reported to the health-care provider are accurate and reliable for patient care decision making. The technical laboratory standards described here provide detailed guidance for performing this validation, including considerations for pre- and postnatal constitutional and neoplastic applications.
Medical laboratory professionals must be prepared to identify, interpret, and report results with clinical relevance while being mindful of the social, ethical, and legal responsibilities of reporting genetic information. The interpretation of the data from microarray analysis into clinically relevant information is a difficult and complex undertaking. No algorithm for copy-number change interpretation can substitute for adequate training and knowledge in the fields of medical genetics, pathology, and oncology. We recommend that CMA analysis be performed in laboratories overseen by individuals with appropriate professional training (i.e., certified by the American Board of Medical Genetics and Genomics [ABMGG] in clinical cytogenetics, clinical molecular genetics or laboratory genetics and genomics or certified by the American Board of Pathology in molecular genetic pathology) and that the interpretation and reporting of clinical microarray findings be performed by these same certified individuals.

Received: 4 May 2021; Revised: 4 May 2021; Accepted: 4 May 2021;

Published online: 15 June 2021

\section{REFERENCES}

1. Manning, M. \& Hudgins, L. Array-based technology and recommendations for utilization in medical genetics practice for detection of chromosomal abnormalities. Genet. Med. 12, 742-745 (2010).

2. Miller, D. T. et al. Consensus statement: chromosomal microarray is a first-tier clinical diagnostic test for individuals with developmental disabilities or congenital anomalies. Am. J. Hum. Genet. 86, 749-764 (2010).

3. Committee on Genetics and the Society for Maternal-Fetal Medicine. Committee Opinion No. 682: Microarrays and next-generation sequencing technology: the use of advanced genetic diagnostic tools in obstetrics and gynecology. Obstet. Gynecol. 128, e262-e268 (2016).

4. Cherry, A. M. et al. Diagnostic cytogenetic testing following positive noninvasive prenatal screening results: a clinical laboratory practice resource of the American College of Medical Genetics and Genomics (ACMG). Genet. Med. 19, 845-850 (2017).

5. Arber, D. A. et al. The 2016 revision to the World Health Organization classification of myeloid neoplasms and acute leukemia. Blood. 127, 2391-2405 (2016).

6. Chun, K. et al. Assessing copy number aberrations and copy-neutral loss-ofheterozygosity across the genome as best practice: An evidence-based review from the Cancer Genomics Consortium (CGC) working group for chronic lymphocytic leukemia. Cancer Genet. 228-229, 236-250 (2018).

7. Dougherty, M. J., Tooke, L. S., Sullivan, L. M., Hakonarson, H., Wainwright, L. M. \& Biegel, J. A. Clinical utilization of high-resolution single nucleotide polymorphism based oligonucleotide arrays in diagnostic studies of pediatric patients with solid tumors. Cancer Genet. 205, 42-54 (2012).

8. Kanagal-Shamanna, R. et al. Assessing copy number aberrations and copy neutral loss of heterozygosity across the genome as best practice: an evidence based review of clinical utility from the Cancer Genomics Consortium (CGC) working group for myelodysplastic syndrome, myelodysplastic/myeloproliferative and myeloproliferative neoplasms. Cancer Genet. 228-229, 197-217 (2018).

9. Wang, Y., Miller, S., Roulston, D., Bixby, D. \& Shao, L. Genome-wide singlenucleotide polymorphism array analysis improves prognostication of acute lymphoblastic leukemia/lymphoma. J. Mol. Diagn. 18, 595-603 (2016).

10. $\mathrm{Xu}, \mathrm{X}$. et al. Assessing copy number abnormalities and copy-neutral loss-ofheterozygosity across the genome as best practice in diagnostic evaluation of acute myeloid leukemia: an evidence-based review from the Cancer Genomics Consortium (CGC) myeloid neoplasms working group. Cancer Genet. 228-229, 218-235 (2018).

11. Roth, J. J. et al. Diagnostic application of high resolution single nucleotide polymorphism array analysis for children with brain tumors. Cancer Genet. 207, 111-123 (2014).

12. Mikhail, F. M. et al. Technical laboratory standards for interpretation and reporting of acquired copy-number abnormalities and copy-neutral loss of heterozygosity in neoplastic disorders: a joint consensus recommendation from the American College of Medical Genetics and Genomics (ACMG) and the Cancer Genomics Consortium (CGC). Genet. Med. 21, 1903-1916 (2019).

13. South, S. T., Lee, C., Lamb, A. N., Higgins, A. W. \& Kearney, H. M. ACMG standards and guidelines for constitutional cytogenomic microarray analysis, including 
postnatal and prenatal applications: revision 2013. Genet. Med. 15, 901-909 (2013).

14. Cooley, L. D., Lebo, M., Li, M. M., Slovak, M. L. \& Wolff, D. J. American College of Medical Genetics and Genomics technical standards and guidelines: microarray analysis for chromosome abnormalities in neoplastic disorders. Genet. Med. 15, 484-494 (2013)

15. Kearney, H. M., South, S. T., Wolff, D. J., Lamb, A., Hamosh, A. \& Rao, K. W. American College of Medical Genetics recommendations for the design and performance expectations for clinical genomic copy number microarrays intended for use in the postnatal setting for detection of constitutional abnormalities. Genet. Med. 13, 676-679 (2011).

16. Lee, C., lafrate, A. J. \& Brothman, A. R. Copy number variations and clinical cytogenetic diagnosis of constitutional disorders. Nat. Genet. 39, S48-54 (2007).

17. Riggs, E. R. et al. Towards an evidence-based process for the clinical interpretation of copy number variation. Clin. Genet. 81, 403-412 (2012).

18. Sayers, E. W. et al. Database resources of the National Center for Biotechnology Information. Nucleic. Acids. Res. 40, D13-D25 (2012).

19. Firth, H. V. et al. DECIPHER: Database of Chromosomal Imbalance and Phenotype in Humans Using Ensembl Resources. Am. J. Hum. Genet. 84, 524-533 (2009).

20. Zhang, J., Feuk, L., Duggan, G. E., Khaja, R. \& Scherer, S. W. Development of bioinformatics resources for display and analysis of copy number and other structural variants in the human genome. Cytogenet. Genome Res. 115, 205-214 (2006).

21. Riggs, E. R. et al. Technical standards for the interpretation and reporting of constitutional copy-number variants: a joint consensus recommendation of the American College of Medical Genetics and Genomics (ACMG) and the Clinical Genome Resource (ClinGen). Genet. Med. 22, 245-257 (2020).

22. van Heesch, S. et al. Systematic biases in DNA copy number originate from isolation procedures. Genome Biol. 14, R33 (2013).

23. Mason-Suares, H. et al. Density matters: comparison of array platforms for detection of copy-number variation and copy-neutral abnormalities. Genet. Med. 15, 706-712 (2013)

24. Conlin, L. K. et al. Mechanisms of mosaicism, chimerism and uniparental disomy identified by single nucleotide polymorphism array analysis. Hum. Mol. Genet. 19, 1263-1275 (2010).

25. Valli, R., Maserati, E., Marletta, C., Pressato, B., Lo Curto, F. \& Pasquali, F. Evaluating chromosomal mosaicism by array comparative genomic hybridization in hematological malignancies: the proposal of a formula. Cancer Genet. 204, 216-218 (2011).

26. Markello, T. C. et al. Sensitive quantification of mosaicism using high density SNP arrays and the cumulative distribution function. Mol. Genet. Metab. 105, 665-671 (2012).

27. Rowsey, R., Znoyko, I. \& Wolff, D. J. Whole-genome single nucleotide polymorphism microarray for copy number and loss of heterozygosity analysis in tumors. Methods Mol. Biol. 1908, 89-111 (2019).
28. Dugoff, L., Norton, M. E. \& Kuller, J. A. The use of chromosomal microarray for prenatal diagnosis. Am. J. Obstet. Gynecol. 215, B2-B9 (2016).

29. Vanakker, O. et al. Implementation of genomic arrays in prenatal diagnosis: the Belgian approach to meet the challenges. Eur. J. Med. Genet. 57, 151-156 (2014).

30. Sahoo, T. et al. Prenatal diagnosis of chromosomal abnormalities using arraybased comparative genomic hybridization. Genet. Med. 8, 719-727 (2006).

31. Filges, I. et al. aCGH on chorionic villi mirrors the complexity of fetoplacental mosaicism in prenatal diagnosis. Prenat. Diagn. 31, 473-478 (2011).

32. Gu, S., Jernegan, M., Van den Veyver, I. B., Peacock, S., Smith, J. \& Breman, A. Chromosomal microarray analysis on uncultured chorionic villus sampling can be complicated by confined placental mosaicism for aneuploidy and microdeletions. Prenat. Diagn. 38, 858-865 (2018).

33. Yong, P. J., McFadden, D. E. \& Robinson, W. P. Developmental origin of chorionic villus cultures from spontaneous abortion and chorionic villus sampling. J. Obstet. Gynaecol. Can. 33, 449-452 (2011).

34. Li, M. M. et al. Points to consider for reporting of germline variation in patients undergoing tumor testing: a statement of the American College of Medical Genetics and Genomics (ACMG). Genet. Med. 22, 1142-1148 (2020).

35. Pinto, D. et al. Comprehensive assessment of array-based platforms and calling algorithms for detection of copy number variants. Nat. Biotechnol. 29, 512-520 (2011).

36. Rehder, C. W., David, K. L., Hirsch, B., Toriello, H. V., Wilson, C. M. \& Kearney, H. M. American College of Medical Genetics and Genomics: standards and guidelines for documenting suspected consanguinity as an incidental finding of genomic testing. Genet. Med. 15, 150-152 (2013).

\section{ACKNOWLEDGEMENTS}

B.A.S. was an ABMGG laboratory genetics and genomics postdoctoral fellow at Duke University Health System and a trainee member of the ACMG Laboratory Quality Assurance Committee during the preparation of this document.

\section{COMPETING INTERESTS}

All members of this workgroup are directors of clinical laboratories that use chromosomal microarray technologies.

\section{ADDITIONAL INFORMATION}

Correspondence and requests for materials should be addressed to ACMG.

Reprints and permission information is available at http://www.nature.com/ reprints

Publisher's note Springer Nature remains neutral with regard to jurisdictional claims in published maps and institutional affiliations. 